\title{
Average evoked responses and psychophysical performance in patients with pseudohypoparathyroidism
}

\author{
MONTE BUCHSBAUM, CATHY KING, AND ROBERT I. HENKIN
}

From the Unit of Psychophysiology, Laboratory of Psychology, and Adult Psychiatry Branch, National Institute of Mental Health, and Section on Neuroendocrinology, Experimental Therapeutics Branch, National Hearent and Lung Institute, National Institutes of Health, Public Health Service, U.S. Department of Health, Education $\mathbb{\mathbb { B }}$ and Welfare, Bethesda, Maryland, U.S.A.

SUMMARY Perceptual function in eight patients with pseudohypoparathyroidism (PHP) wast studied with two neurophysiological measures, the average evoked response (AER) and nerve्? conduction velocity. A battery of psychophysical tasks including reaction time, size estimation 'hidden pictures,' and the rod and frame procedure was also used. Patients with PHP had significantly longer latency visual AER and slower reaction time than a group of normal volunteers, and they performed erratically and poorly on the other psychophysical tasks. Differentiating thes patients with diffuse mental deficiency were two relatively specific perceptual response patterns: 0 (1) AER amplitude decreased with increasing stimulus intensity, and (2) reaction time was ab옥 normally influenced by the duration of the preparatory interval. Both of these patterns have pe्eviously been found in other patient groups.

Pseudohypoparathyroidism (PHP) is a rare metabolic disorder in which kidney and bone are relatively resistant to parathyroid hormone. This hormone regulates calcium and phosphorus metabolism and decreases renal tubular reabsorption of phosphorus. With diminished parathyroid function, renal tubular reabsorption of phosphorus increases and serum phosphorus rises; concomitantly, hypocalcaemia may result. In PHP, administration of parathyroid extract sufficient to decrease renal tubular reabsorption of phosphorus in normal subjects and in patients with hypoparathyroidism does not produce this effect, demonstrating end organ resistance (Mann, Alterman, and Hills, 1962). Physical features of patients with this disease include brachydactyly, round facies, short stature, and obesity; in addition, these patients exhibit mental deficiency, hyposmia, elevated thresholds for the tastes of sour and bitter (Henkin, 1968), and abnormal electroencephalograms (EEG) (Frame and Carter, 1955; Rose and Vas, 1966). When serum calcium is low patients may exhibit tetany or seizures which may relate to hyperexcitability of their peripheral and central nervous systems. The finding of multiple cases in a single family (Dickson, Morita, Cowsert, Grave and Meyer, 1960) and hyposmia in the mothers of some of these patients (Henkin, 1968) suggest familial factors in PHP. While some improve ment of mental status may result when patients

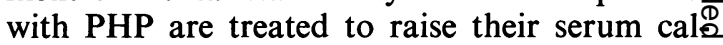
cium, we have observed little or no change in $\vec{B}$ sensory function (Henkin, 1968). In contrast to these sensory findings, the abnormal electro encephalograms return to or toward normal on correction of the serum calcium (Frame and Carter, 1955; Rose and Vas, 1966).

The average evoked response (AER), which is obtained by averaging the EEG responses tó many repetitions of a stimulus, reflects both neurophysiological processes and sensory funco tion. In this technique, the irregular background activity from unrelated neural and scalp muscle. processes is averaged out, whereas specific repetitive electrical activity resulting from the stimulus becomes clearly defined. AER can be्ల recorded from subjects by presenting any sensory stimulus-for example, light flashes, clicks of tones-which has an onset abrupt enough t\& produce synchrony and repeatability of brain response. 
Since we have previously found specific sensory and neural defects associated with several endocrinopathies (Henkin, 1967; Ojemann and Henkin, 1967; Henkin, 1968; Henkin and Daly, 1968), the administration of a battery of neurosensory and perceptual tasks seemed useful in the present study. The contradiction between our finding of the association of short latency evoked responses with low serum calcium concentrations (Buchsbaum and Henkin, 1970) and reports of long latency AER in mental defectives (Hogan and Graham, 1967; Nodar and Graham, 1968) further suggested the potential usefulness of AER techniques.

\section{METHODS}

Eight patients with PHP (four males and four females aged 13 to 48 years) were studied. Clinical data are summarized in Table 1 . Hospitalized at the NIH Clinical Center in an air-conditioned metabolic

TABLE 1

SUMMARY OF CLINICAL DATA

\begin{tabular}{|c|c|c|c|c|c|}
\hline Patient & $\begin{array}{l}\text { Age } \\
(y r)\end{array}$ & $\begin{array}{c}\text { Serum } \\
\text { Ca } \\
(\mathrm{mg} / 100 \mathrm{ml} .)\end{array}$ & $\begin{array}{c}\text { Mean } \\
\text { AER } \\
\text { latency* } \\
\text { (msec) }\end{array}$ & $\begin{array}{c}\text { Mean } \\
\text { nerve } \\
\text { conduction } \\
\text { velocity } \\
(\mathrm{m} / \mathrm{sec})\end{array}$ & $I Q$ \\
\hline B.A. $\dagger$ & 25 & $9 \cdot 3$ & 172 & 61 & 56 \\
\hline S.B. & 14 & $8 \cdot 6$ & 133 & 64 & 61 \\
\hline E.D. & 18 & $9 \cdot 8$ & 181 & 51 & (60) \\
\hline B.H. & 17 & $7 \cdot 8$ & 160 & 76 & 102 \\
\hline M.J.† & 48 & $8 \cdot 2$ & 145 & 47 & 83 \\
\hline A.M. $\dagger$ & 13 & 8.6 & 155 & 65 & (60) \\
\hline M.M. & 18 & $8 \cdot 5$ & 118 & 66 & 90 \\
\hline K.U. & 28 & $9 \cdot 0$ & 151 & 63 & 62 \\
\hline
\end{tabular}

$*=\mathrm{N}_{120}$.

$\dagger=$ Basal ganglia calcification.

Parentheses $=$ estimate of IQ.

ward, the patients, who all had clinical features and laboratory findings of PHP, ate a diet containing $400 \mathrm{mg}$ calcium and $800 \mathrm{mg}$ phosphorus and drank a constant amount of fluid. Serum calcium concentrations were obtained in each patient throughout the study and reported for the day in which the AER latency was performed. Patients received from 400 to 3,600 units of parathyroid extract administered intramuscularly, and all exhibited resistance evidenced by less than normal responses of serum and urinary calcium and phosphorus. Radiographs of the skull were obtained in each patient. Six patients received daily vitamin $\mathrm{D}$, usually 50,000 units taken orally, but serum calcium concentration remained low. No patient exhibited tetany or seizure activity during the study. Some studies of these patients have been described previously (Zisman, Henkin, Ross, and Bartter, 1970). Eighty paid college students (40 males and 40 females, aged 18 to 31 ) served as normal volunteers.

AER TECHNIQUE The subject sat in a darkened room in front of a diffuse light source. Four intensities of light flashes were presented in randomized blocks of 10. The interval between individual stimuli was one second. Each intensity of light was presented 120 times for a total of 480 stimuli. Grass disk electrodes were used to record the EEG between vertex and right ear with left ear at ground potential. The amplified EEG was computer-summed separately for each intensity for $500 \mathrm{msec}$ after the light flash. Measurements of the amplitudes and latencies of the major sequence of positive, negative, and positive peaks $\left(\boldsymbol{P}_{80}, N_{120}, \mathrm{P}_{200}\right)^{1}$ were made. A straight line function was fitted, with the least squares technique, to the amplitude-light intensity and latency-light intensity data. This procedure is described in detail elsewhere (Buchsbaum and Silverman, 1968).

NERVE CONDUCTION VELOCITY TECHNIQUE Ulnar motor nerve conduction velocity was measured in each patient by a modification of the Helmholtz technique (Eaton and Lambert, 1957; Henkin, Gill, Warmolts, Carr, and Bartter, 1963) and compared with similar measurements made on 36 normal volunteers of similar age and sex.

PERCEPTUAL TASKS A battery of tasks selected to sample different dimensions of perceptual response was administered to seven of the patients. One patient (B.A.) was unable to complete the test battery because of poor eyesight. Twenty of the normal volunteers served as controls.

1. Rod and frame test (Witkin, Dyk, Faterson, Goodenough, and Karp, 1962). Seated in front of an apparatus which consisted of a luminous rod within a luminous square frame, in an otherwise totally darkened room, the subject was required to adjust the rod so that it was vertical-that is, parallel with the walls of the room. The test was scored as the mean degree of deviation from vertical for eight trials, in degrees.

2. Disk size estimation (Silverman, 1964). A circular spot of light was displayed on a small rearprojection screen. The subject's task was to adjust the size of the light spot by means of a hand crank until it matched the size of one of the three standard circular disks. The disks were presented in four

1 Subscripts refer to the time in milliseconds, after the flash, at which the wave appears; $P$ refers to a positive peak; $N$, a negative peak. 
consecutive series of three trials with a random order of disk sizes $(4.92 \mathrm{~cm}, 4.77 \mathrm{~cm}, 5.00 \mathrm{~cm})$. An accuracy score was computed in terms of the mean deviation, in millimetres, of the adjusted light spot from the actual disk size.

3. Kinaesthetic figural after-effects (KFA) (Buchsbaum and Silverman, 1968). A blindfolded subject stood between a horizontal bar of uniform width on his right and a comparison bar graduated in $\frac{1}{16}$ in. steps of width on his left. The subject made four judgments ('pre-stimulation') of the place on the graduated bar which he felt was just as wide as the uniform bar. The subject then moved to two other bars over which he rubbed his fingers simultaneously at a rate of approximately three times per second for 30 seconds ('stimulation'). After this he returned to the original bars and made an equivalence judgment as before ('post-stimulation'). This sequence of stimulation followed by equivalence judgment was repeated four times. Scores were computed as the mean deviation in inches by which post-stimulation judgments differed from pre-stimulation judgments.

4. Reaction time (Zahn and Rosenthal, 1965). The subject sat before a telegraph key and a loudspeaker. He was told to depress the key at a signal from the experimenter and to release it as soon as possible after the onset of a tone. Before each trial, the experimenter said 'ready' and the subject then depressed the key, initiating a delay which served as a pre-set preparatory interval. Ten trials each of five preparatory intervals $(1,2,4,7$, and 15 seconds) were administered. After a two minute break, the subject was given an irregular series of preparatory interval trials in which the same five intervals were used but in random order. The results were reported as change in reaction time with increasing preparatory interval duration and variability. The 'Set Index' (Rodnick and Shakow, 1940), an empirical discriminating formula designed to separate responses of schizophrenic from normal patients, was also computed.

5. Hidden pictures (Thurstone, 1944). The first part of the test required the patient to find as many faces as he could in three black and white pictures with faces hidden in the background, and was scored for number of faces correctly identified. The second part of the test required the patient to give specific information about the picture in which the faces were hidden ('incidental noticing'). These results were scored for the number of questions answered correctly.

6. Intelligence quotients (IQ) These were obtained by several different techniques including the Weschsler Adult Intelligence Scale.

\section{RESULTS}

AER RESUlts 1. Latency Patients with PHP had significantly longer evoked response latencies than did the normal volunteers. Mean latency for all four intensities were significantly longer ( $t$ test, $\mathrm{P}<0.02$ ) for peaks $\mathrm{N}_{120}$ and $\mathrm{P}_{200}$. Three patients with PHP had latencies more than two standard deviations above the mean of the normal volunteers.

2. Effect of light intensity Patients with PHP showed significantly less decrease in latency with increasing stimulus intensity $(t$ test on latencyintensity slopes for $\left.\mathrm{N}_{120}, \mathrm{P}<0.05\right)$ than did the normals (Fig. 1). The patients also showed reduction in amplitude with increasing stimulus

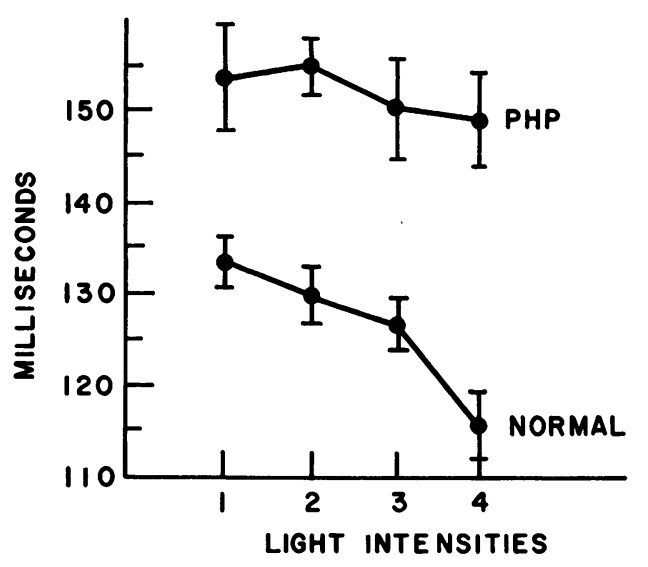

FIG. 1. Average evoked response latency for peak $N_{120}$ (in msec, on the ordinate) for four light intensities (corresponding to settings 1, 2, 4, and 16 on a Grass Model PS 2 photostimulator, on the abscissa) for normal volunteers and patients with PHP. Normal subjects show a decrease in latency as light intensity increases. In contrast, patients with PHP change latency slightly with increasing light intensity.

intensity for peak $\mathrm{N}_{120}$ with a mean slope of $-0.60 \mu \mathrm{v} / \log$-lumen sec. This was significantly different from the normals ( $t$ test, $\mathrm{P}<0.05$; normal males $\bar{X}=-0 \cdot 14$, normal females $\bar{X}=$ $+0 \cdot 46)$.

3. Effect of serum calcium concentration Patients with PHP with serum calcium concentrations greater than or equal to $9 \mathrm{mg} / 100 \mathrm{ml}$. had a mean latency for $\mathrm{N}_{120}$ of $174 \mathrm{msec}$; patients 
with serum calcium concentrations less than $9 \mathrm{mg} / 100 \mathrm{ml}$. had a mean latency of $142 \mathrm{msec}$.

4. Effect of $I Q$ No statistically significant correlation between IQ and mean AER latency was found $(r=-0 \cdot 33, P>0 \cdot 05)$. Although most patients with PHP have mental deficiency, they represent a heterogeneous population, and there were patients with normal IQs yet prolonged AER latency (Table 1). However, patients with IQ less than 70 had mean latency values for $\mathrm{N}_{120}$ of $161 \mathrm{msec}$, whereas patients with IQs over 80 had a mean latency of 141 msec. The normal group, with a mean IQ of 120 , had a mean latency of $138 \mathrm{msec}$. These results suggest that low IQ was generally associated with long AER latency.

5. Nerve conduction velocity The mean ulnar motor nerve conduction velocity in the patients with PHP was $62 \mathrm{~m} / \mathrm{sec}$ which does not differ significantly from that observed in normal volunteers $(56 \mathrm{~m} / \mathrm{sec})$. However the range of the conduction velocities of the patients was much wider $(47 \mathrm{~m} / \mathrm{sec}-76 \mathrm{~m} / \mathrm{sec})$ than that observed in the volunteers $(52 \mathrm{~m} / \mathrm{sec}-68 \mathrm{~m} / \mathrm{sec})$. It was not correlated with their IQ, reaction time, or serum calcium concentration.

6. Cerebral calcifications and AER Three of the patients had calcifications of the basal ganglia (Table 1). Five had calcifications of the dura mater, falx cerebri, or choroid plexus. Each of the three patients with basal ganglia calcifications had negative AER slopes. The patients with the densest basal ganglia calcification had the most negative slope in the group. In contrast, three of the five patients without calcifications had positive AER slopes. No correlation with AER latency or IQ was observed.

PSYCHOPHYSICAL RESULTS Patients with PHP showed a wide range of response scores on the perceptual tasks, often performing outside both the upper and lower limits of the control group. Scores on all tests tended to reflect more inaccurate performance than that shown by the control group (Table 2).

Rod and frame test Patients with PHP adjusted the rod significantly further from the vertical than the normal volunteers. Only one subject (B.H.) scored within the range of the normal volunteers.
TABLE 2

TEST SCORES

\begin{tabular}{lcccc}
\hline \multicolumn{1}{c}{ Task } & \multicolumn{2}{c}{$P$ PP patients } & \multicolumn{2}{c}{ Normals } \\
\hline & $\bar{X}$ & SD & $\bar{X}$ & SD \\
Rod and frame & $23.60^{\circ}$ & 24.8 & $5 \cdot 2^{\circ}$ & 11.9 \\
Disk size estimation & $5.44 \mathrm{~mm}$ & 1.3 & $5.22 \mathrm{~mm}$ & 0.5 \\
KFA & $-0.20 \mathrm{in}$. & 0.39 & $-0.13 \mathrm{in}$. & $0 \cdot 15$ \\
Reaction time (1 sec) & $517.0 \mathrm{msec}$ & 248.30 & $178.0 \mathrm{msec}$ & $10 \cdot 50$ \\
Set index & 1319.0 & 187.0 & 413.0 & 112.0 \\
Hidden faces & 3.5 & 4.9 & 9.4 & $5 \cdot 7$ \\
Incidental noticing & 3.5 & 4.4 & 9.5 & 7.9 \\
& & & & \\
\hline
\end{tabular}

Disk size estimation The patients generally overestimated the disk size but the mean did not differ significantly from that of the normal volunteers. The range of the PHP patients' scores, $15 \mathrm{~mm}$, was three times that observed in the normal volunteers.

$K F A$ The patients generally underestimated the size of the bar after stimulation, but their mean score did not differ significantly from that of the normal volunteers; however, the range of scores, almost $1 / 2$ in., was twice that of the normal volunteers.

Reaction time The patients had significantly slower reaction times than did the normal volunteers. For the one second preparatory interval reaction time there was no overlap between the groups. The most severely retarded subjects made the slowest and most erratic reaction times. The Set Index also separated the two groups without overlap. No significant correlations with either IQ or serum calcium concentration were found. The data for the regular and irregular series are presented in Fig. 2. The rate of change in reaction time as a function of increasing duration of preparatory interval was expressed as the slope of the line fitting the mean reaction time-log preparatory interval data (least squares method). For normal volunteers the observed slopes ( 4.6 and 0.0 for the regular and irregular series respectively) are close to those observed for normals by Zahn and Rosenthal (3.9 and -1.2) and show the same divergence of the regular and irregular series that they observed (Zahn and Rosenthal, 1965) in a group of schizophrenic patients $(6.0$ and $-17 \cdot 8)$.

\section{DISCUSSION}

While the poor and erratic perceptual perform- 


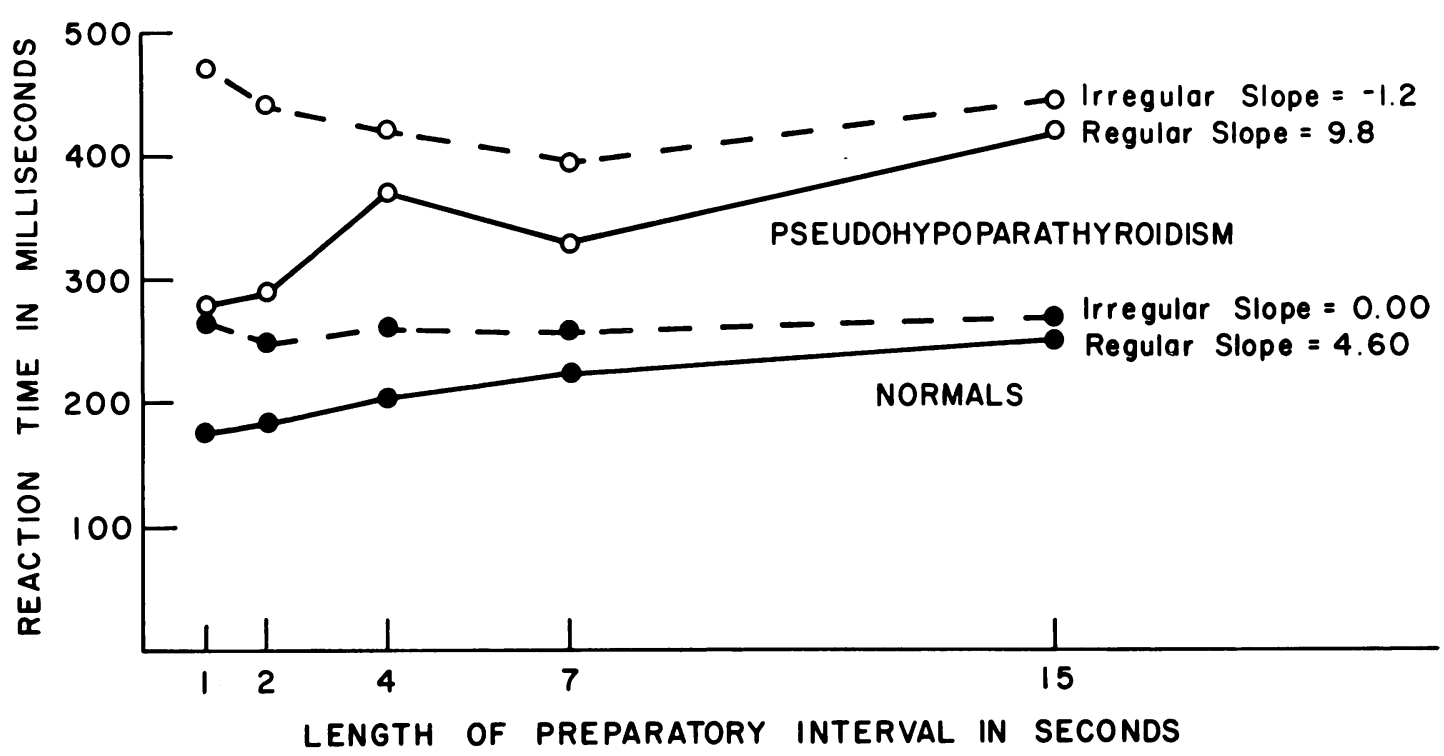

FIG. 2. Reaction time for normals and patients with PHP. Normal subjects show small increase in reaction $\omega_{\tilde{N}}^{\omega}$ time with increasing duration of regular preparatory intervals (slope 4.60) and little decrease with increasing iv duration of irregular preparatory intervals (slope 0.0). In contrast, patients with PHP show strong effects of the N duration of the preparatory interval.

ance shown by the patients with PHP might largely be expected from patients with mild to moderate intellectual deficiency, two measuresthe reaction time and AER slope-suggest more specific deficits. We observed the same difference in reaction time response pattern between normals and patients with PHP as did Zahn and Rosenthal (1965) between normal subjects and patients with acute schizophrenia. Of significance are the greater increases in reaction time with increasing length of preparatory interval in the patient as compared with the control group in the regular series and the greater decrease in reaction time in the irregular series. The greater increase in reaction time with increasing length of preparatory interval in the regular series appears to reflect the greater difficulty the patients with PHP had in maintaining a mental set over a long interval. Zahn and Rosenthal explained the decrease in reaction time with increasing length of the preparatory interval in the irregular series as a function of the preceding preparatory interval. Reaction time trials with one second preparatory intervals were usually preceded by trials with longer preparatory intervals. After a long preparatory interval trial, subjects generally are not quite as ready to respond in the first few seconds of the next preparatory interval as they tend to expees⿱⺈.⺕․ another long preparatory interval. Norma $\overrightarrow{0}$ subjects in our sample, as in that of Zahn and Rosenthal, were able to respond to the signal tone with minimal effects of the irrelevant information provided by the last preparatory interval. However, this ability was not found in patients with schizophrenia (Zahn and Rosenthal, 1965) or with PHP. Indeed, patients with mental deficiencies with the same range of IQ as patients with PHP clearly resembled normal subjects on an essentially identical procedure (Tizard and Venables, 1956); the mean reaction time in the patients with mental defectiveness did not differ from normal. On a similar visual reaction time task Costa found that a group of patients with various cerebral diseases showed slow reaction times that did not change with preparatory interval (Costa, 1962). Since patients with mental defectiveness exhibited normal reaction times and brain-damaged patients did not show the preparatory interval effect exhibited by patients with PHP the abnormal performance of patients with PHP must reflect something other than low IQ or non-specific cerebral damage.

If some simple physiological factor such as nerve conduction velocity, muscle tone, or 
sense organ deficit were responsible for the slow reaction times observed in our subjects we would expect to see the normal preparatory intervalreaction time curves merely shifted upward. Instead, we observed additionally an increase in the difference in the effect of preparatory interval between the regular and irregular series-evidence that higher levels of processing must be involved in the poor performance of patients with PHP.

The AER results in patients with PHP also parallel an earlier reported finding of decreasing AER amplitude with increasing stimulus intensity in a group of nonparanoid schizophrenic patients (Buchsbaum and Silverman, 1968). This phenomenon has been called 'reduction' (Buchsbaum and Silverman, 1968; Buchsbaum and Pfefferbaum, 1971), and is relatively more common in men than women (Buchsbaum and Pfefferbaum, 1971). The association of 'reducing' in men and in patients with PHP and basal ganglia calcification is interesting, since this same association has been observed in a small series of patients with Parkinson's disease (unpublished data).

The observed decrease in AER latency in the patients with lower than normal serum calcium concentration is consistent with our earlier findings that AER latency decreases with decreasing serum calcium concentration (Buchsbaum and Henkin, 1970). It also suggests that the general lengthening of AER latency in patients with PHP is due not to hypocalcaemia but rather to some other manifestation of CNS changes in this disease. The basis of the relationship between changes in motor nerve conduction velocity, AER amplitude and latency, and serum calcium is not clear. The observed increase in AER amplitude at the rate of approximately $1 \mu \mathrm{v} / \mathrm{mg} / 100 \mathrm{ml}$. decrease in serum calcium concentration (between 6 and $8 \mathrm{mg} / 100 \mathrm{ml}$.) (Buchsbaum and Henkin, 1970) cannot be clearly related to the effects of extracellular calcium concentration on resting membrane potential or excitability. The maximum observed change in serum calcium concentration in our patients, a decrease of about $25 \%$, appears to be too small to effect any significant change in ion or membrane potential phenomena as noted from in vitro data from other investigators (Shanes, 1958). Further, low extracellular calcium concentrations are usually associated with smaller spike potentials rather than larger ones. While the relationship between AER and spike potentials is quite uncertain, it is difficult to reconcile the opposite direction of these effects.

The observed decrease in AER latency (Buchsbaum and Henkin, 1970) at the rate of approximately $10 \mathrm{msec} / \mathrm{mg} / 100 \mathrm{ml}$. decrease in serum calcium concentration (between 6 and $8 \mathrm{mg} / 100 \mathrm{ml}$.) may be more closely related to the manner by which changes in calcium metabolism affect multi-synaptic systems than to any effect they may have on changes in axonal conduction. Given conduction of the order of 45 to $60 \mathrm{~m} / \mathrm{sec}$ and the relatively short distance $(10$ to $20 \mathrm{~cm})$ between retina and cortex, delays of $100 \mathrm{msec}$ cannot be explained by changes in conduction speed alone. It is well known that calcium ions are essential for normal synaptic transmission (Feng, 1936; Del Castillo and Stark, 1952) but their main effect is not on the nerve per se, but rather on the neuromuscular junction (Dodge and Rahamimoff, 1967).

Not previously taken into consideration is the possible role of adenosine $3^{\prime}, 5^{\prime}$-monophosphate (cyclic AMP) in the AER and perceptual changes observed in patients with PHP for they have lower than normal urinary excretion of cyclic AMP (Chase, Melson, and Aurbach, 1969). Although these measurements have not been made in patients with schizophrenia, significant alterations in urinary excretion of cyclic AMP have been observed in patients with some affective disorders (Paul, Cramer, and Bunney, 1971). These latter patients have been observed to exhibit various AER changes (Buchsbaum, Goodwin, Murphy, and Borge, 1971).

Specific neurophysiological or perceptual deficits are often overlooked in describing clinical syndromes. The approach used here may not only offer diagnostic clues about the disease but also provide new ways of approaching the perceptual processes themselves.

\section{REFERENCES}

Buchsbaum, M., and Henkin, R. I. (1970). Serum calcium concentration and the average evoked response. Electroencephalography and Clinical Neurophysiology, 30, 10-16.

Buchsbaum, M., and Pfefferbaum, A. (1971). Individual differences in stimulus intensity control. Psychophysiology, 8, 600-611.

Buchsbaum, M., and Silverman, J. (1968). Stimulus intensity control and the cortical evoked response. Psychosomatic Medicine, 30, 12-22.

Buchsbaum, M., Goodwin, F., Murphy, D., and Borge, G. (1971). AER in affective disorders. American Journal of Psychiatry, 128, 19-25.

Chase, L. R., Melson, G. L., and Aurbach, G. D. (1969). Pseudohypoparathyroidism: Defective excretion of $3^{\prime}, 5^{\prime}-$ 
AMP in response to parathyroid hormone. Journal of Clinical Investigation, 48, 1832-1844.

Costa, L. D. (1962). Visual reaction time of patients with cerebral disease as a function of length and constancy of preparatory interval. Perceptual and Motor Skills, 14, 391-397.

Del Castillo, J., and Stark, L. (1952). The effect of calcium ions on the motor end-plate potentials. Journal of Physio$\log y, 116,507-515$.

Dickson, L. G., Morita, Y., Cowsert, E. J., Graves, J., and Meyer, J. S. (1960). Neurological, electroencephalographic, and heredofamiliar aspects of pseudohypoparathyroidism and pseudo-pseudohypoparathyroidism. Journal of Neurology, Neurosurgery, and Psychiatry, 23, 33-39.

Dodge, F. A., and Rahamimoff, R. (1967). Cooperative action of calcium ions in transmitter release at the neuromuscular junction. Journal of Physiology, 193, 419-432.

Eaton, L. M., and Lambert, E. H. (1957). Electromyography and electric stimulation of nerves in diseases of motor unit. Journal of the American Medical Association, 103, 11171124.

Feng, T. P. (1936). Studies on the neuromuscular junction. II. The universal antagonism between calcium and curarizing agencies. Chinese Journal of Physiology, 10, 513-527.

Frame, B., and Carter, S. (1955). Pseudohypoparathyroidism. Neurology (Minneap.), 5, 297-310.

Henkin, R. I. (1967). Abnormalities of taste and olfaction in patients with chromatin negative gonadal dysgenesis. Journal of Clinical Endocrinology and Metabolism, 27, 1436-1440.

Henkin, R. I. (1968). Impairment of olfaction and of the tastes of sour and bitter in pseudohypoparathyroidism. Journal of Clinical Endocrinology and Metabolism, 28, 624-628.

Henkin, R. I., and Daly, R. L. (1968). Auditory detection and perception in normal man and in patients with adrenal cortical insufficiency: Effect of adrenal cortical steroids. Journal of Clinical Investigation, 47, 1269-1280.

Henkin, R. I., Gill, J. R., Jr., Warmolts, J. R., Carr, A. A., and Bartter, F. C. (1963). Steroid-dependent increase of nerve connection velocity in adrenal insufficiency. Journal of Clinical Investigation, 42, 941.

Hogan, D. D., and Graham, J. T. (1967). The use of a summing computer for analyzing auditory evoked responses of mentally retarded adults. Journal of Auditory Research, 7, 1-13.
Mann, J. B., Alterman, S., and Hills, A. G. (1962). Albright's hereditary osteodystrophy comprising pseudohypoparathyroidism and pseudopseudohypoparathyroidism. Annals of Internal Medicine, 56, 315-342.

Nodar, R. H., and Graham, J. T. (1968). An investigation of auditory evoked responses of mentally retarded adults during sleep. Electroencephalography and Clinical Neurophysiology, 25, 73-76.

Ojemann, G. A., and Henkin, R. I. (1967). Steroid dependent changes in human visual evoked potentials. Life Sciences, 6, 327-334.

Paul, M. I., Cramer, H., and Bunney, W. E., Jr. (1971). Urinary adenosine $3^{\prime}, 5^{\prime}$-monophosphate in the switch process from depression to mania. Science, 171, 300-303.

Rodnick, E. H., and Shakow, D. (1940). Set in the schizophrenic as measured by a composite reaction time index. American Journal of Psychiatry, 97, 214-225.

Rose, G. A., and Vas, C. J. (1966). Neurological complications and electroencephalographic changes in hypoparathyroidism. Acta Neurologica Scandinavica, 42, 537-550.

Shanes, A. M. (1958). Electrochemical aspects of physiological and pharmacological action in excitable cells. Part II. The action potential and excitation. Pharmacological Reviews, 10, 165-273.

Silverman, J. (1964). Scanning-control mechanism and 'cognitive filtering' in paranoid and non-paranoid schizophrenia. Journal of Consulting Psychology, 28, 385-393.

Thurstone, L. L. (1944). A Factorial Study of Perception. University of Chicago Press: Chicago.

Tizard, J., and Venables, P. H. (1956). Reaction time responses by schizophrenics, mental defectives and normal adults. American Journal of Psychiatry, 112, 803-807.

Witkin, H., Dyk, R. B., Faterson, H. F., Goodenough $\Omega$ D. R., and Karp, S. A. (1962). Psychological Differentia $=$ tion: Studies of Development. Wiley: New York.

Zahn, T. P., and Rosenthal, D. (1965). Preparatory set iro acute schizophrenia. Journal of Nervous and Menta? Disease, 141, 352-358.

Zisman, E., Henkin, R. I., Ross, G. T., and Bartter, F. C. (1970). A biochemical similarity between chromatin negative gonadal dysgenesis and pseudohypoparathyroidism: Resistance to parathyroid extract. Acta Endocrinologica Panamericana, 1, 49-71. 